\title{
Micromechanical testing of SU-8 cantilevers
}

\author{
M. HOPCROFT ${ }^{1}$, T. KRAMER ${ }^{2}$, G. KIM ${ }^{2,4}$, K. TAKASHIMA ${ }^{3}$, Y. HIGO ${ }^{3}$, D. MOORE ${ }^{1}$ and J. BRUGGER ${ }^{2}$ \\ ${ }^{1}$ Department of Engineering, Cambridge University, Cambridge CB2 1PZ, U.K., ${ }^{2}$ Microsystems Laboratory, Ecole Polytechnique Fédérale de Lausanne, \\ CH-1015 Lausanne, Switzerland, ${ }^{3}$ P. \& I. Laboratory, Tokyo Institute of Technology, 4259 Nagatsuta, Midori-ku, Yokohama 226-8503, \\ Japan, ${ }^{4}$ Present address: School of Mechanical Engineering, Kyungpook National University, Daegu 702-701, Korea
}

Received in final form 14 September 2004

\begin{abstract}
SU-8 is a photoplastic polymer with a wide range of possible applications in microtechnology. Cantilevers designed for atomic force microscopes were fabricated in SU-8. The mechanical properties of these cantilevers were investigated using two microscale testing techniques: contact surface profilometer beam deflection and static load deflection at a point on the beam using a specially designed test machine. The SU-8 Young's modulus value from the microscale test methods is approximately $2-3 \mathrm{GPa}$.
\end{abstract}

Keywords AFM cantilevers; microscale mechanical test; SU-8; surface profliometer; Young's modulus.

\section{INTRODUCTION}

The field of microtechnology and Micro-electromechanical systems (MEMS) has grown rapidly for the past two decades. ${ }^{1}$ Recently, researchers are moving away from the traditional silicon-based technologies and investigating alternative materials. One of the promising candidate materials for new microsystems is the polymer SU-8. ${ }^{2}$ SU-8 has useful material properties, including photosensitivity, transparency to visible light, a low Young's modulus and biocompatibility.

In order to design MEMS structures using SU-8, accurate material property data must be available. However, it is difficult to apply the traditional macroscale mechanical test methods to microscale samples, so reliable micromechanical property data are scarce. In this paper, we present the design and fabrication of cantilevers for commercial atomic force microscopes (AFM) constructed from SU-8. These cantilevers are tested using two different microscale methods to measure the stiffness of the cantilever and to determine the Young's modulus of the SU-8 material. The test results indicate that existing macroscale material test methods are not sufficiently precise for MEMS design purposes and that reliable microscale test methods are required.

\section{SU-8 CANTILEVERS FOR ATOMIC FORCE MICROSCOPES}

Atomic force microscopes have become an essential tool for surface analysis. In an AFM, a sharp tip attached to a

Correspondence: J. Brugger. E-mail: juergen.brugger@epfl.ch cantilever is scanned over the sample surface. The cantilever is deflected by features on the surface, and the deflection is detected using a laser which is reflected off the beam. Figure 1 illustrates the principle of AFM operation to produce a profile of the sample surface with subnanometer resolution.

For effective operation, an AFM requires a cantilever with specific properties. It must have a high resonance frequency $(>10 \mathrm{kHz})$ and a low stiffness $\left(0.1-1 \mathrm{~N} \mathrm{~m}^{-1}\right)$. Using the conventional silicon-based microfabrication techniques, it is difficult to make a cantilever with a sufficiently low spring constant to achieve the small forces that are required, as the thinness of the cantilever material becomes a limiting factor. Photoplastic polymers with a low Young's modulus (below $\sim 5 \mathrm{GPa}$ ) are an interesting possible alternative, because they can be used to fabricate thick, robust cantilevers with the required properties. ${ }^{3}$

One of these polymers is the negative photoresist SU-8. With a highly cross-linked structure, it is thermally and chemically stable allowing the realization of thick microstructures with high aspect ratios. The use of SU-8 also enables a simpler, cheaper and more versatile cantilever fabrication process based on surface micromachining. This makes the integration of additional functionality easier and it allows a variety of cantilever shapes to be designed. For example, SU-8 has recently been used to fabricate cantilevers with integrated piezoresistive sensors. ${ }^{4}$

The near-UV negative photoresist SU-8 is based on the EPON ${ }^{\circledR}$ Resin SU-8 from Resolution Europe BV (Hoogvliet, The Netherlands). Layer thicknesses ranging from $750 \mathrm{~nm}$ to $300 \mu \mathrm{m}$ can be realized with spin-coating techniques, and structures of up to $2 \mathrm{~mm}$ in height can 


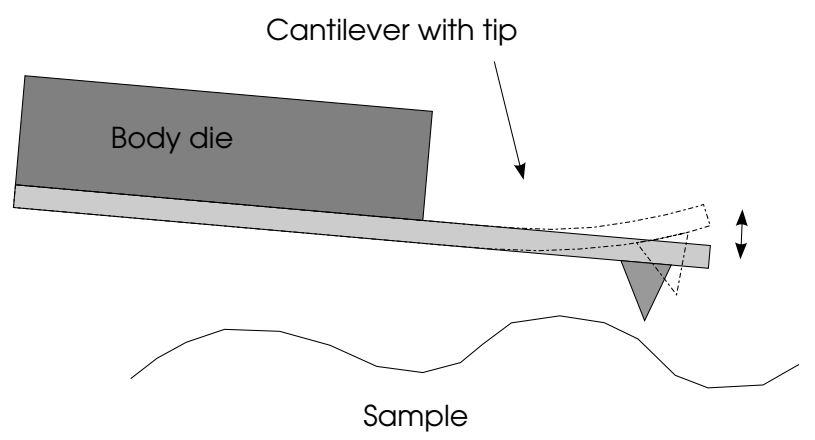

Fig. 1 Schematic illustration of atomic force microscope (AFM) operation.

be made by applying multiple coats. ${ }^{2}$ High-aspect ratio structures are fabricated using thick SU-8 and standard lithography tools. With its advantageous chemical and mechanical properties and compatibility with the standard lithography, SU-8 is a promising candidate for MEMS applications, including packaging, micromoulds and microchannels. $^{5}$

The complete SU-8 cantilever chip consists of a cantilever attached to a large body die, and the fabrication process is illustrated in Fig. 2. The procedure begins with deposition of a sacrificial layer of $200 \mathrm{~nm}$ Cr followed by $500 \mathrm{~nm} \mathrm{Al}$ onto a silicon substrate. The first layer of SU-8 (Sotec 60/40) is then spun on and the cantilever pattern is exposed with UV lithography but not developed. ${ }^{6,7}$ The second layer of SU-8 (Sotec 70/30) is then spun on and the body patterns are exposed. Then, all the unexposed SU-8 is developed and removed. Finally, the sacrificial layer is etched and the cantilever chips are removed from the substrate. To simplify the release of the cantilevers from the substrate, the different chips are attached to a SU-8 frame, which holds the chips together. The individual cantilever chips are then mechanically detached from the frame as needed. Figure 3 shows a scanning electron microscope (SEM) image of a fabricated cantilever chip.

The cantilevers were designed with thickness $5 \mu \mathrm{m}$, width $55 \mu \mathrm{m}$ and a range of lengths between $250 \mu \mathrm{m}$ and $475 \mu \mathrm{m}$. The body die contains alignment features which were designed to match the alignment features used in a commercial AFM manufactured by Nanosurf AG (Liestal, Switzerland). ${ }^{8}$ The cantilevers shown here do not include an AFM tip.

\section{SURFACE PROFILOMETER FOR MICROSCALE MECHANICAL TESTING}

Micromechanical property data are needed for the efficient design of microsystems, but traditional macroscale mechanical test methods are difficult to apply to microscale materials due to the small size and fragility of the samples. Recent reports in the literature have focused (a)

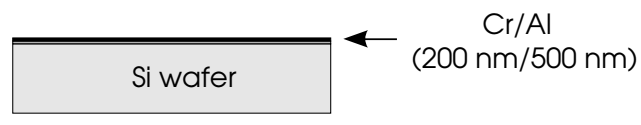

(b)

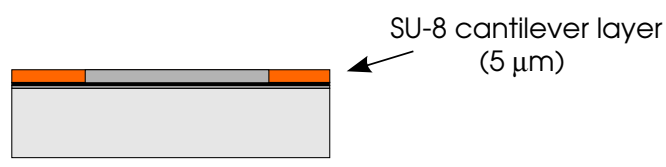

(C)

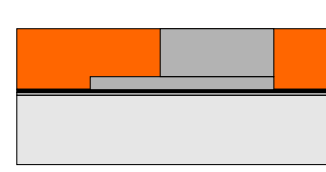

SU-8 body layer

$(280 \mu \mathrm{m})$

(d)

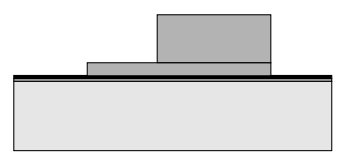

SU-8 development

(e)

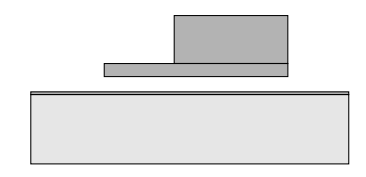

Release of chips

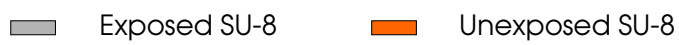

Fig. 2 Schematic illustration of the fabrication process for SU-8 cantilevers: (a) $\mathrm{Cr} / \mathrm{Al}$ sacrificial layer is deposited on the silicon substrate; (b) a $5 \mu \mathrm{m}$ layer of SU-8 is spun on and exposed to define the cantilevers; (c) a $280 \mu \mathrm{m}$ layer of SU-8 is spun on and exposed to define the body; (d) the SU-8 layers are developed and (e) the sacrificial layer is etched and the cantilever chips are removed.

on the development of new microscale mechanical test methods. ${ }^{9-11}$ Many test methods are specific to certain classes of material, typically involve extensive fabrication procedures, are subject to errors, and may require expensive test instruments. A new test method, called MATTest, which addresses these issues has recently been developed. ${ }^{12,13}$ MAT-Test (an acronym for materials testing) achieves improvements in test quality through the use of a contact surface profilometer as the test instrument. The testing approach is applicable to a wide range of thin film materials, requires only simple test structures to be fabricated, is insensitive to potential test errors, and involves a profilometer which is a widely available laboratory test instrument.

The MAT-Test procedure is illustrated in Fig. 4. A contact surface profilometer stylus is scanned along the length of a suspended cantilever beam fabricated from the material under test. The stylus contacts the sample with a constant force, the cantilever beam is deflected downwards by the stylus, and this is recorded as the instrument traces along the beam. This data set can be analysed in combination with the geometry of the test structure to determine 


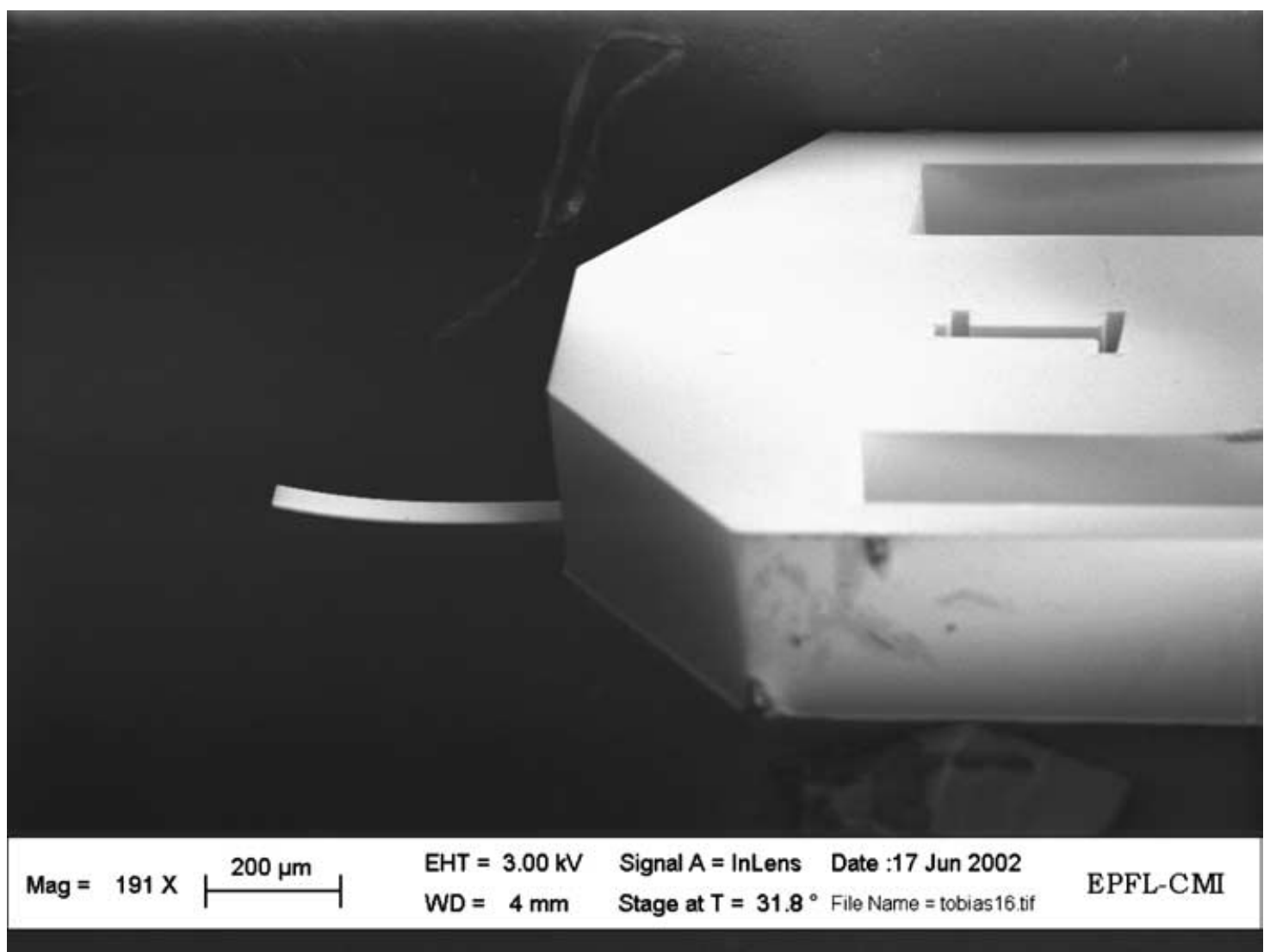

Fig. 3 Scanning electron microscope (SEM) image of microfabricated SU-8 cantilever.

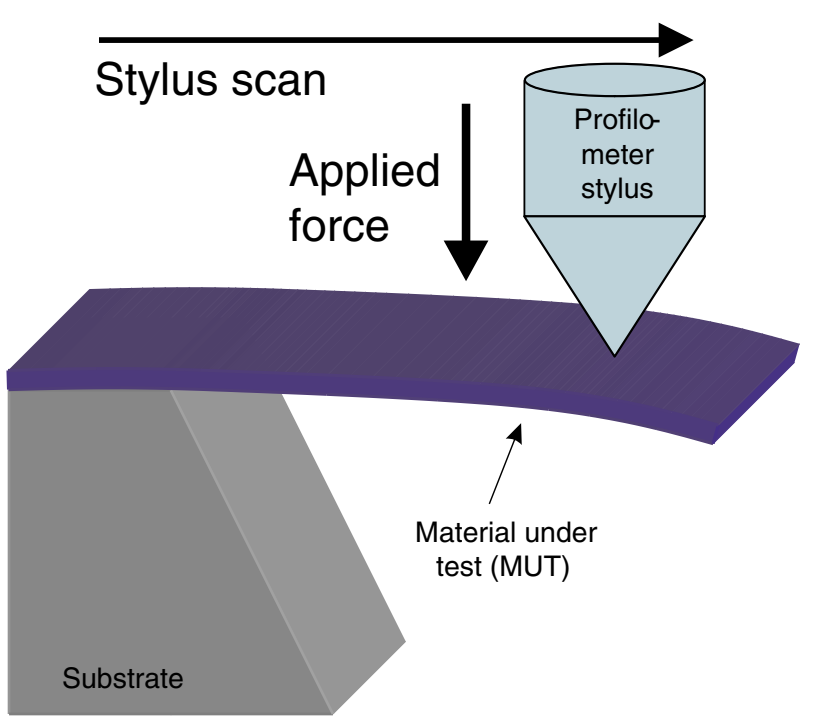

Fig. 4 Schematic illustration of MAT-Test procedure. ${ }^{11}$

the Young's modulus of the material under test in a manner that is insensitive to potential errors in test structure fabrication, such as undercut of the cantilever root or residual stress in the material under test. ${ }^{12}$
This test method is easy to perform and is widely applicable to material systems in which suspended cantilever structures can be fabricated. It applies to most common material combinations currently used in microsystems, i.e. a thin film deposited on a substrate such as silicon nitride on silicon. It can also be applied to more exotic materials, such as spin-coated polymers.

The MAT-Test experiments on SU-8 cantilevers described in this paper used a Dektak 3ST contact surface profilometer. ${ }^{14}$ Figure 5 shows a microphotograph of a cantilever being scanned in the profilometer.

The first requirement for accurate Young's modulus data is to establish that the cantilever is being deflected only in its elastic region, and that no plastic deformation is occurring. Figure 6 shows the results of five successive measurements of the same cantilever plotted on the same figure. The sample orientation is shown in Fig. 5, with the base of the cantilever at the left, and the sample was scanned from left to right. The small region of positive slope at the start of the cantilever is a topographical feature of the sample and does not represent bending of the cantilever. The measurements show no significant change from one to the other, indicating that the material is not plastically deformed by the profilometer scan. Note that 


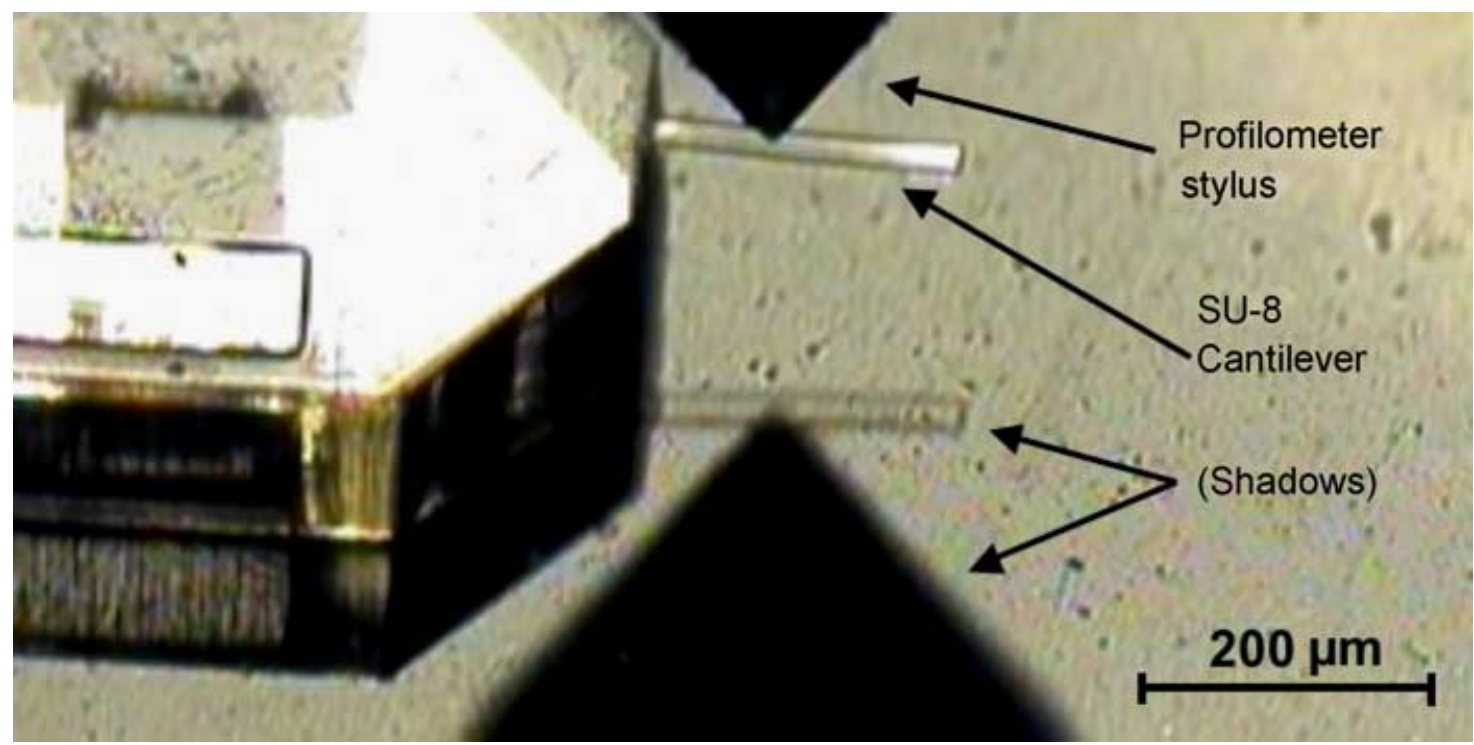

Fig. 5 Optical microscope image showing SU-8 cantilever under test.

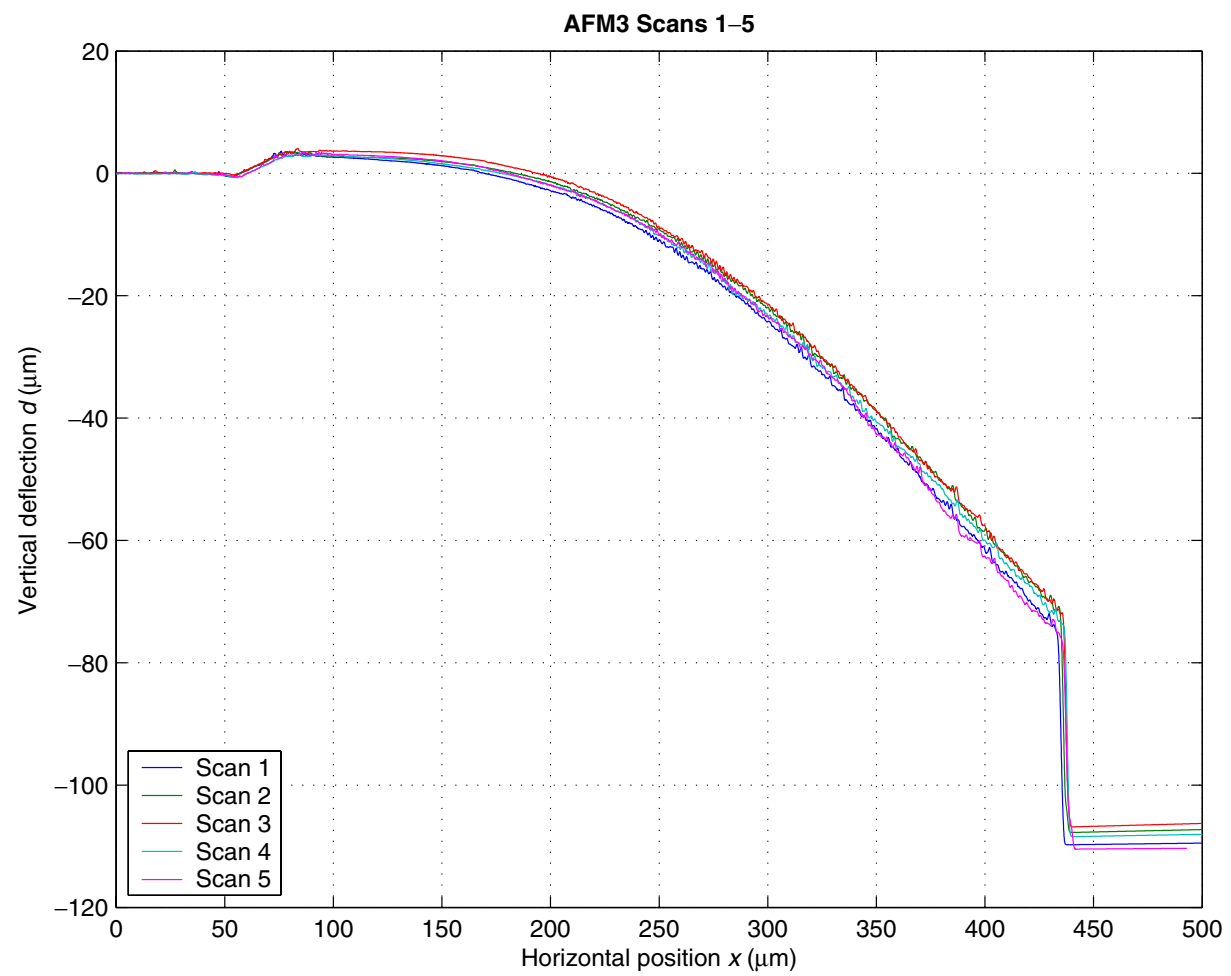

Fig. 6 Graphical representation of vertical deflection as function of lateral tip position for repeated deformation of SU-8 cantilevers.

this procedure could be used to determine the yield stress of the material, simply by increasing the applied force until deformation is observed.

Next, the data are analysed. Figure 7 shows a screenshot of the MAT-Test data analysis software, which was written in the MATLAB. ${ }^{15}$ The software displays the profilometer scan data in the upper plot, and the operator selects the rel- evant region of the data for analysis, as shown in the lower plot. In the case of the SU-8 cantilevers, this selection step is important because the cantilevers are very compliant and even the smallest available profilometer force causes the far end of the cantilever to enter the large deflection regime. The most straightforward analysis is when deflection data are confined to the small deflection regime and 


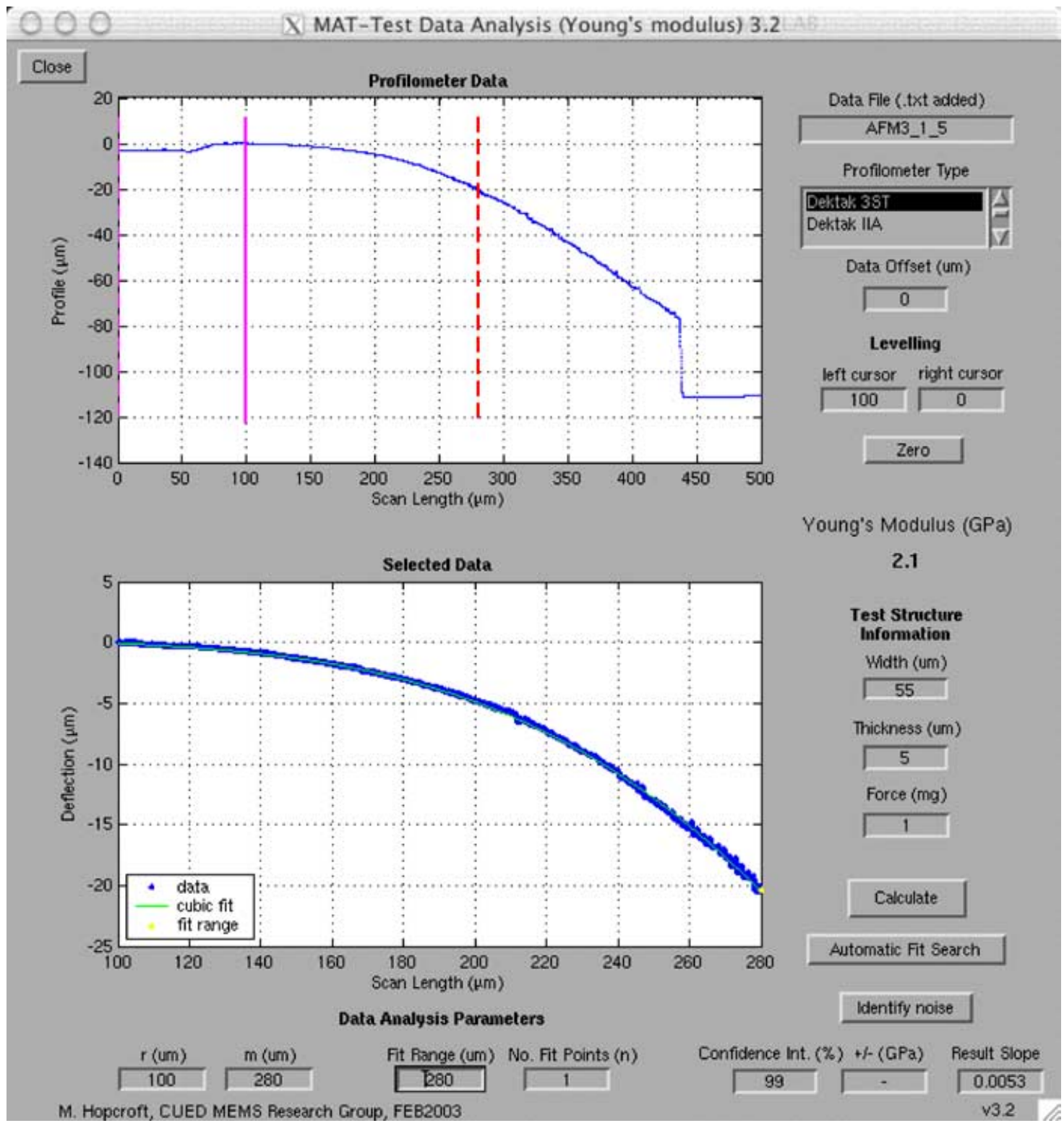

Fig. 7 MAT-Test analysis software.

the sample deflection is described by the Euler equation: ${ }^{1}$

$d=F L^{3} /(3 E I)$,

where $d$ is deflection of the cantilever, $F$ is the applied force, $L$ is the length of the cantilever, $E$ is the Young's modulus of the cantilever material and $I$ is the second moment of area of the cantilever cross-section. The Euler small deflection analysis yields acceptable results when the ratio of cantilever deflection to cantilever length, $d / L$, is less than approximately 0.1 .

The fitting procedure for the deflection is based upon finding the coefficient of the cube of the distance along the microcantilever. Any initial bending near the root of the cantilever or imperfect levelling of the sample during profiling only contributes to the linear coefficient of distance along the cantilever. For singly supported cantilevers of material with a stress gradient through the thickness of the cantilever, the relaxed state of the beam is the arc of a circle. Such stress gradients only contribute to the coefficient of the square of the distance along the microcantilever in this case where the deflections are small and all these effects are additive.

Using the fitting procedure based upon the cubic coefficient, the average Young's modulus result from the SU-8 cantilevers using the MAT-Test technique is $2.7 \pm$ $0.5 \mathrm{GPa}$. The accuracy of the method depends upon the force calibration of the profilometer, the noise in the data 


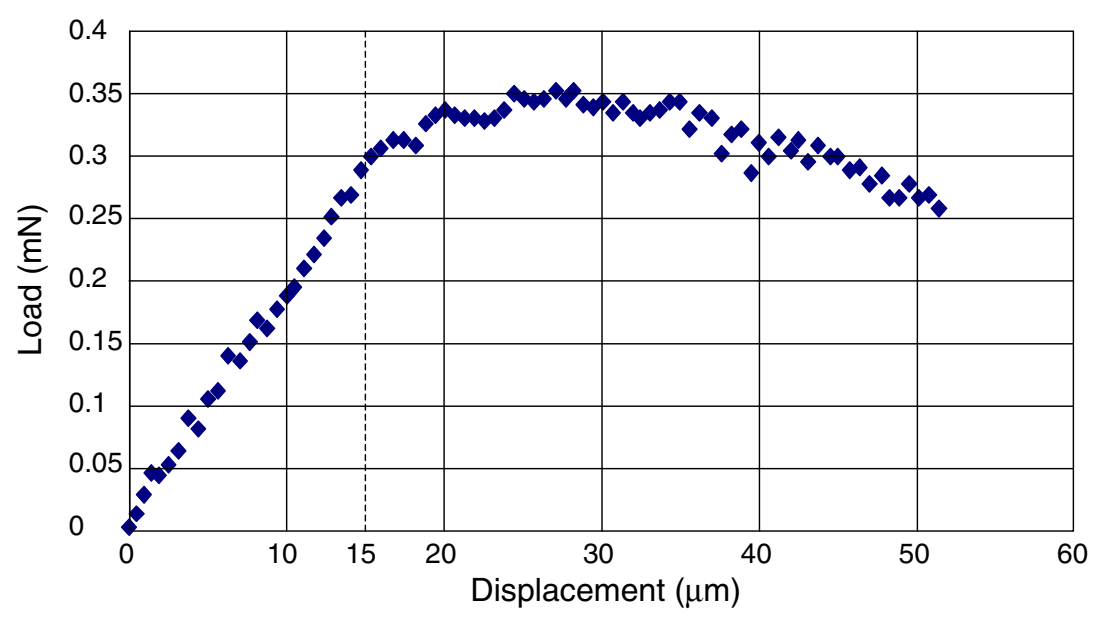

Fig. 8 Load-displacement curve for SU-8 cantilever loading.

when fitting to find the cubic coefficient and the precision of the cantilever geometry measurement.

\section{MICROSCALE MECHANICAL TEST MACHINE}

Static load bending tests on SU-8 cantilever specimens from the same fabrication batch were carried out using a specialized mechanical testing machine for microsized specimens, MFT2000, which was developed at the Tokyo Institute of Technology. ${ }^{16,17}$ This test machine is capable of applying both static and cyclic loads to microfabricated specimens. Tensile, compression and bending tests can be performed by changing loading and specimen fixtures. The load resolution is $10 \mu \mathrm{N}$ and the displacement resolution is $5 \mathrm{~nm}$. The loading position can be adjusted by a precision $\mathrm{X}-\mathrm{Y}$ stage with a translation resolution of $0.1 \mu \mathrm{m}$. Further details of the testing machine are described elsewhere. $^{16,17}$

Bending tests on the SU-8 cantilevers were performed by loading the cantilevers at a fixed position along the cantilever and recording the resulting deflection. The loading position of the cantilever beam was set at $56 \mu \mathrm{m}$ from the fixed end of the specimen. Static load was applied to the specimen by a diamond tip with a radius of $5 \mu \mathrm{m}$, which was connected directly to the actuator. Figure 8 shows the load-displacement curve obtained from loading the cantilever. A linear relationship between load and displacement is observed up to a displacement of $15 \mu \mathrm{m}$. The bending test was stopped at a displacement of $50 \mu \mathrm{m}$.

The load-displacement curve in Fig. 8 deviates from a linear relationship for displacements greater than $15 \mu \mathrm{m}$. Figure 9 shows a series of photographs taken during the bending test. As seen in Fig. 9d, the position of the diamond tip appears to have slipped along the beam, and the deviation from the linear load-displacement relationship is partially due to this slipping. It is interesting to note that the specimen was not broken even after a bending displacement of $50 \mu \mathrm{m}$ as shown in Fig. 9d. Figure 9e shows the cantilever after the bending test. The specimen exhibited slight plastic deformation after removal of the load but no cracking was observed with laser microscope inspection.

From the linear portion of the experimental loaddisplacement curve in Fig. 8, the Young's modulus was calculated to be $1.9 \pm 0.5 \mathrm{GPa}$. The measurement precision is determined by the accuracy of the measurements of the specimen dimensions, the loading tip position relative to the root of the cantilever and the force.

\section{DISCUSSION}

The Young's modulus test results with the two test methods are comparable and give approximately 2-3 GPa for these microfabricated SU-8 structures. The values are somewhat lower than other data in the literature. ${ }^{1}$ This may be due to differences in fabricated SU-8 structures according to the solvent removal and cross-linking process conditions. Materials such as SU-8 may behave differently on the microscale compared with larger samples because the solvent removal is more efficient through the surfaces of small structures. Furthermore, the material properties of many thin-film materials vary considerably depending on their deposition and curing conditions, necessitating mechanical testing of realistic MEMS structures.

The results of this study highlight the need for reliable microscale material test methods for MEMS research and design. For example, the initial batch of AFM cantilevers had approximately half the intended spring constant, because macroscale test data had been used in the design process.

Development of MAT-Test is ongoing and only preliminary results have been presented. In cases where the 

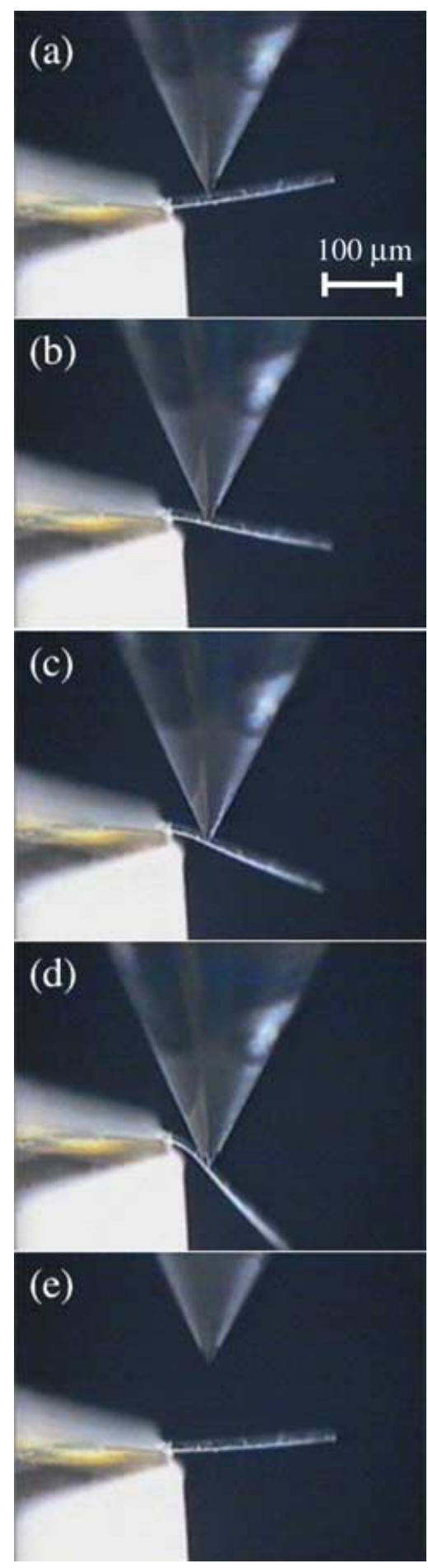

Fig. 9 Optical microscope image of SU-8 cantilevers being tested.

profilometer is capable of taking two traces of the same beam with different contact forces, it can be advantageous to calculate the difference between the two measurements before fitting these data to find the cubic coefficient. The SU-8 cantilevers tested in this study are not stiff and the profilometer had to be used near the limits of the resolution of the test equipment. However, the results are instructive, both for the results of the test and for the test method. Previous experiments on $5 \mu \mathrm{m}$ thick $\mathrm{SiC}$ cantilevers with a larger Young's modulus were more straightforward. ${ }^{12}$

Another well-established procedure for obtaining mechanical data and Young's modulus is by making resonance frequency measurements in vacuum. ${ }^{1}$ However, the profilometry approach is convenient and can be extended to testing beams to destruction and measuring materials limits. Not all contact surface profilometer mechanisms are capable of applying a constant force to the sample as the beam is displaced. To facilitate the use of such instruments it would be useful to develop standard calibration samples, for example sets of single crystal silicon microbeams with a range of different widths.

\section{CONCLUSION}

The fabrication of AFM cantilevers was used as an example of MEMS applications of SU-8 to illustrate the potential usefulness of SU-8 in a broad range of MEMS from cantilevers to fluid microchannels. Two microscale material property test methods were applied to SU-8. A surface profilometer approach (MAT-Test) involves a standard contact profilometer to scan along microbeams to perform a convenient test that can be applied to a variety of materials. The MFT2000 test machine is a more specialized but versatile instrument that is used to perform a variety of mechanical tests on a microscale samples. Microscale test methods such as these must be introduced and certified as standards, enabling MEMS device designers' devices to use known material specifications. This will facilitate more widespread, reliable and robust MEMS design for commercial products.

\section{Acknowledgement}

The authors gratefully acknowledge experimental collaboration with Dr. M. Ahmad, Imperial College, London.

\section{REFERENCES}

1 Gad-el-Hak, M. (2002) MEMS Handbook. CRC Press, Boca Raton, FL, USA. ISBN 0-8493-0077-0.

2 Lorenz, H., Despont, M., Fahrni, N., Lablance, N., Renaud, P. and Vettiger, P. (1997) A low cost negative resist for MEMS. $\mathcal{F}$. Micromech. Microeng. 7, 121-124.

3 Genolet, G., Brugger, J., Despont, M. et al. (1999) Soft, entirely photoplastic probes for scanning force microscopy. Rev. Sci. Instrum. 70, 2398-2401.

4 Thaysen, J., Yalçinkaya, A. D., Vestergaard, R. K. et al. (2002) SU8-based piezoresistive mechanical sensor. In: Micro Electro Mechanical Systems 2002 (IEEE), Kyoto, Japan, pp. 320-323. 
5 Despont, M., Lorenz, H., Fahrni, N., Brugger, J., Renaud, P. and Vettiger, P. (1997) Micro Electro Mechanical Systems, 1997 (IEEE), Nagoya, Japan, pp. 518-522.

6 Kramer, T. (2002) Realization of self-alignment cantilever chips based on SU-8. Semester Project in Microsystems Lab (LMIS1), EPFL Lausanne, Switzerland.

7 SOTEC Microsystems. (2002) Available at http://www.somisys.ch/microclips.html.

8 Nanosurf AG. (2002) Available at http://www.nanosurf.com/.

9 Schweitz, J.-A. (1992) Mechanical characterization of thin films by micromechanical techniques. MRS Bulletin 17, 34-45.

$10 \mathrm{Kraft}$, O. and Volkert, C. A. (2001) Mechanical testing of thin films and small structures. Adv. Engng Mater. 3, 99110 .

11 Osterberg, P. M. and Senturia, S. D. (1997) M-TEST: A test chip for MEMS material property measurement using electrostatically actuated test structures. F. Microelectromech. Syst. 6, 107-118.

12 Hopcroft, M. A. (2002) MAT-Test: A new method for thin film materials characterization. MPhil Thesis, Engineering Department, Cambridge University, UK.

13 Moore, D. F., Williams, J. A., Hopcroft, M. A., Boyle, P., He, J. H. and Syms, R. R. A. (2003) Laser micromachining of thin film for optoelectronic devices and packages. In: Laser Micromachining for Optoelectronic Packages (SPIE), Brugges, Belgium, pp. 140-147.

14 Veeco Dektak (2002) 3ST Surface Profilometer. Available at http://www.veeco.com/.

15 The Mathworks, Inc. (2002) MATLAB. Available at http://www.mathworks.com/matlab/.

16 Higo, Y., Takashima, K., Shimojo, M., Sugiura, S., Pfister, B. and Swain, M. V. (2000) Fatigue testing machine of micro-sized specimens for MEMS applications. Mater. Res. Soc. Symp. Proc. 605, 241-246.

17 Takashima, K., Higo, Y., Sugiura, S. and Shimojo, M. (2001) Fatigue crack growth behavior of micro-sized specimens prepared from an electroless plated $\mathrm{Ni}-\mathrm{P}$ amorphous alloy thin film. Mater. Trans. 42, 68-73. 